\title{
Factores Determinantes de la Productividad Laboral en Pequeñas y Medianas Empresas de Confecciones del Área Metropolitana de Bucaramanga, Colombia
}

\author{
Ludym Jaimes $^{(1) \star}$, Marianela Luzardo(1) y Miguel D. Rojas ${ }^{(2)}$ \\ (1) Universidad Pontificia Bolivariana, Facultad de Ingeniería Industrial, Escuela de Ingenierías, \\ Km. 6 Vía Piedecuesta, Bucaramanga-Colombia (e-mail: ludym.jaimes@upb.edu.co; \\ marianela.luzardo@upb.edu.co) \\ (2) Universidad Nacional de Colombia, Facultad de Minas, Departamento de Ingeniería de la Organización, \\ Carrera 80 no.65-223, Medellín- Colombia (e-mail: mdrojas@unal.edu.co)
}

* Autor a quien debe ser enviada la correspondencia

Recibido Dic. 1, 2018; Aceptado Feb. 12, 2018; Versión final Abr. 12, 2018, Publicado Oct. 2018

\begin{abstract}
Resumen
El objetivo del trabajo presentado en este artículo es identificar los factores determinantes de la productividad laboral en las pequeñas y medianas empresas (PYMES) del sector confecciones del área metropolitana de Bucaramanga en Colombia. El sector confecciones tiene más de cien años de historia aportando a la economía colombiana, y caracterizado por el uso intensivo de mano de obra y la generación de empleo. Las PYMES enfrentan situaciones particulares de competencia por limitaciones en capital humano y económico. En 39 PYMES de la zona se aplicó una encuesta sobre productividad laboral desde dos dimensiones: la humana y la del proceso productivo. Usando el análisis factorial exploratorio se identificó que para la dimensión humana los factores comportamiento grupal y ambiente social de trabajo explican el $62.32 \%$ de la variabilidad de los datos. Los factores más influyentes de la dimensión del proceso productivo son gestión de procesos y capacidades/control, los cuales explican el 59.42\% de la variabilidad total de la dimensión.
\end{abstract}

\section{Determinant Factors of Labor Productivity in Clothing Small and Medium Size Enterprises of the Metropolitan Area of Bucaramanga, Colombia}

\begin{abstract}
The objective of the work presented in this article is to identify the determinant factors of labor productivity in small and medium size enterprises (SMEs) in the clothing sector of the Metropolitan Area of Bucaramanga in Colombia. The clothing sector has more than one hundred years of history, contributing to the Colombian economy, and characterized by the intensive use of labor and the generation of employment. SMEs face particular situations of competition due to limitations in human and economic capital. A survey was applied to 39 SMEs on labor productivity from two dimensions: human and productive process. Using factorial exploratory analysis, it was found that for the human dimension the factors group behavior and social work environment, explain $62.32 \%$ of the variability of the data; The most influential factors of the productive process dimension of the are process management and capacities / control, which explain $59.42 \%$ of the total variability.
\end{abstract}




\section{INTRODUCCIÓN}

La productividad es estudiada por diversas disciplinas; presenta sus orígenes en las ciencias económicas, siendo reconocido Adam Smith, como quien introdujo el concepto en "La riqueza de las naciones". Desde la ingeniería, Satish et al. (2013) plantean que la productividad es en gran medida una función de la toma de decisiones en varios niveles, y debería ser entendida en las organizaciones, caracterizadas en el contexto actual por condiciones de volatilidad, incertidumbre, complejidad, ambigüedad y retroalimentación retardada. Cequea y Rodríguez-Monroy (2012) develan la multidimensionalidad del concepto al enunciar que la productividad es "el resultado de la articulación armónica entre la tecnología, la organización y el talento humano, combinando en forma óptima o equilibrada los recursos para la obtención de los objetivos". Por esto, los gobiernos y empresas propenden por la mejora de la productividad en las organizaciones, Porter (2009) afirma que "El objetivo principal de una nación es conseguir un alto y creciente nivel de vida para sus ciudadanos, la actitud para hacerlo depende de la productividad con la que se empleen la mano de obra y el capital"; de otro lado, el Consejo Privado de Competitividad de Colombia lo reafirma: "el principal desafío que enfrenta el sector privado es el aumento de su productividad" (Consejo Privado de Competitividad, 2016).

La investigación se enfoca en la productividad del factor humano, denominada productividad laboral -PL-, dado su impacto en el desempeño empresarial y el crecimiento económico de las naciones. La PL se calcula como la relación entre las salidas del proceso productivo y la entrada de capital de trabajo (Sumanth, 1996); sin embargo, como indicador clave para medir el desarrollo es tema de interés multidisciplinar, que abarca aspectos productivos y sociolaborales (Oficina Regional de la OIT para América Latina y el Caribe, 2012). El amplio uso del Producto Interno Bruto -PIB- per cápita como medida de crecimiento da relevancia a la PL, mostrando la relación entre productividad y crecimiento; el PIB por habitante, es la medida más sencilla de crecimiento, es el resultado de la PL por la fracción de la población que trabaja.

El artículo presenta los resultados de la identificación de los factores determinantes de la PL en pequeñas y medianas empresas -pymes- del sector confecciones del Área Metropolitana de Bucaramanga -AMB-; lo cual es pertinente ya que éste sector se caracteriza por el uso intensivo de mano de obra, especialmente no calificada (Zuleta y Jaramillo, 1996). Las pymes se encuentran en todos los sectores productivos en Latinoamérica y tienen una creciente importancia en esta región, dado su impacto en el empleo (Cardozo et al., 2011). Las industrias de bajo valor agregado y mano de obra intensiva, como el sector confecciones, tienen un alto potencial de generación de empleo. Por lo tanto, es esencial darles una atención urgente en términos de mejorar la producción, la productividad y la eficiencia (Parida y Pradhan, 2016). Hernández et al. (2017) afirman que las pequeñas empresas para responder a los cambios del entorno tecnológico y alcanzar una productividad de alto nivel deben actuar de forma contundente, considerando aspectos como: la practicidad de los procesos, el uso integral de los sistemas de comunicación, la inversión en actividades de investigación y el desarrollo de una cultura de conocimiento.

Los costos laborales en Colombia son altos, esto estimula el interés por su control, así como por la PL considerando su relación. Se reconoce la dificultad de adopción de estrategias para mejorar la PL en las pymes, debido a diferencias en condiciones de funcionamiento y gestión ante las grandes empresas (Chadwick et al., 2013; Mathur et al., 2012). En Colombia la clasificación de las empresas por tamaño está regida por el artículo 2을 de la Ley 590 de 2000, modificado por el artículo $2^{\circ}$ de la Ley 905 de 2004 (Congreso de la República, 2004). De acuerdo con la planta de personal y el nivel de activos, los tamaños se determinan como detalla la Tabla 1.

Tabla 1: Clasificación de las empresas según el tamaño (Datos tomados de Ley 905 de 2004)

\begin{tabular}{|l|l|l|}
\hline \multicolumn{1}{|c|}{ Tamaño de empresa } & \multicolumn{1}{|c|}{ Planta de personal } & \multicolumn{1}{c|}{ Activos totales } \\
\hline Microempresa & No superior a 10 trabajadores. & $\begin{array}{l}\text { Excluida la vivienda, por valor inferior a } 500 \text { salarios } \\
\text { mínimos mensuales legales vigentes (SMMLV) }\end{array}$ \\
\hline Pequeña empresa & Entre 11 y 50 trabajadores. & Entre 501 y menos de 5.000 SMMLV \\
\hline Mediana empresa & Entre 51 y 200 trabajadores. & Entre 5.001 a 30.000 SMMLV \\
\hline Gran Empresa & Superior a 200 trabajadores. & Superiores a 30.000 SMMLV \\
\hline
\end{tabular}

El sector confecciones de Bucaramanga y su área metropolitana impacta de manera significativa la economía a nivel nacional, ubicándose en quinto lugar en cuanto a cantidad de establecimientos que participan en la encuesta anual manufacturera, representa el $4,2 \%$ del total de empresas del sector (DANE, 2016); sobresale la actividad del sector en la elaboración de prendas de vestir infantiles, por esto se desarrolla en el AMB desde 
1996 la Feria EIMI -Exposición internacional de Moda Infantil-, evento que genera actividad comercial significativa con participación de empresarios y comerciantes de índole nacional e internacional.

El sector confecciones en el departamento de Santander cuenta con 1745 empresas, según registros de la Cámara de Comercio de Bucaramanga de 2017(Cámara de Comercio de Bucaramanga, 2017), de las cuales el 92,2\% están ubicadas en el AMB. La importancia del sector, junto con las necesidades particulares de las pymes, la multidimensionalidad de la productividad y la baja PL del sector en el AMB muestran la relevancia y pertinencia de esta investigación, con el objetivo de identificar los factores determinantes de la PL en pymes de confecciones del AMB, y así abrir caminos para su gestión en las organizaciones, aportando a la competitividad empresarial y al desarrollo regional.

El estudio de la productividad laboral desde dos dimensiones importantes: la humana y la de los procesos, llevó a identificar como factores determinantes de la PL para la primera dimensión: el comportamiento grupal y ambiente social de trabajo; y para la segunda: la gestión de los procesos y las capacidades/control de la empresa. Los resultados son la base para concluir sobre la necesidad de incentivar en los empresarios la importancia de capacitar al personal, promover y facilitar su participación en la mejora del proceso productivo y dar relevancia a contar con información sobre aspectos psicosociales del personal.

\section{CONTEXTO DEL SECTOR CONFECCIONES}

El sector confecciones se caracteriza por ser gran empleador; cuenta con cerca de 10.000 empresas a nivel nacional, representando el $9,3 \%$ del PIB manufacturero colombiano y el $1 \%$ del PIB nacional; junto con el sector textil en 2013 ocupó a 580.000 personas (Ministerio de Comercio, Industria y Turismo, 2013). El sector vincula aproximadamente el $10 \%$ del personal ocupado en la industria colombiana, dato que se evidencia en la encuesta anual manufacturera (DANE, 2016).

El sector es importante a nivel nacional; "para el siglo XXI el desempeño económico se puede describir en dos fases, para el período 2001-2006, la tasa promedio de crecimiento del sector textil-confección fue de $4,6 \%$, comportamiento por encima de la economía colombiana, $4,1 \%$. Sin embargo, para los años posteriores 2007-2013 el desempeño del sector no ha sido alentador debido a factores externos como la crisis económica y financiera a nivel mundial" (SURA, 2014).

El sector confecciones en Colombia como sector de tradición, con más de 100 años de historia industrial ha sido considerado en diversos análisis de competitividad nacional. A inicios de los 90's el estudio del grupo consultor Monitor incluyó el sector confecciones, reconociéndolo como gran empleador nacional. En 2008 el Gobierno Nacional creó el programa de transformación productiva, con el fin de mejorar la competitividad de la economía colombiana, el programa agrupa los sectores en dos categorías: nuevos o emergentes y más y mejor de lo bueno; en esta última se encuentra el sector confecciones. Los informes de gestión del programa plantean como principal brecha del sector, la gestión de la productividad; en el informe de 2013 se estableció como trabajo futuro para el sector la implementación de programas de gestión de la productividad empresarial y laboral en pro de desarrollar capacidades para flexibilizar la estructura de producción y mejorar su rentabilidad (Ministerio de Comercio, Industria y Turismo, 2013).

\section{FACTORES DETERMINANTES DE LA PRODUCTIVIDAD LABORAL}

La PL o productividad del factor humano, y su impacto en el desempeño empresarial, son estudiados desde diferentes enfoques y disciplinas. Desde la economía, Adam Smith (1776) afirma que el producto anual de la tierra y del trabajo de la nación solo puede incrementarse por dos vías: un adelanto en las facultades productivas del trabajo útil que dentro de ellas se mantiene, o el aumento en la cantidad del trabajo. Desde la ingeniería, la PL se concibe como el resultado del sistema productivo generado por el desempeño de las personas en un lugar de trabajo y está asociado con la optimización de los recursos materiales, financieros y tecnológicos de la organización (Cequea y Rodríguez-Monroy, 2012). Desde la administración la productividad se considera un índice clave para evaluar el desempeño empresarial y su mejora un objetivo organizacional, el cual con el paso del tiempo toma mayor relevancia (Yang y Su, 2012).

La productividad es relevante, Porter (2009) afirma: "la productividad es el determinante fundamental del nivel de vida de una nación a largo plazo... El nivel de vida de una nación depende de la capacidad de sus empresas para lograr altos niveles de productividad y para aumentar ésta a lo largo del tiempo". Ahora bien, la amplitud de literatura del tema evidencia la creciente importancia del capital humano en las economías, donde el conocimiento es una de las principales fuentes de ventajas competitivas (Davide et al., 2010). Durante un largo período fue común considerar al trabajador como sólo otro factor de producción a explotar en las empresas para obtener la máxima utilización (Putnik et al., 2012); sin embargo, otra línea de pensamiento valora y resalta el rol del personal en la mejora de la productividad (Fahed-Sreih, 2012). 
Los hallazgos de la revisión de literatura incluyen diversas investigaciones en términos de PL, dando evidencia de aportes en diferentes sectores, sobresaliendo la cantidad de estudios del tema en el sector de la construcción, dada su importancia en las economías nacionales. Aunque el enfoque de la investigación es el sector confecciones, algunos elementos explorados en la construcción pueden ser transferidos a las confecciones dada la similitud entre los dos sectores en cuanto al uso intensivo de mano de obra, fundamentalmente de carácter operativo. En el sector construcción, Rojas y Aramvareekul (2003) establecen tres categorías asociadas a la PL: sistemas de gestión y estrategias; mano de obra; medio ambiente de la industria y condiciones externas; identificando las dos primeras como determinantes. Las variables consideradas en la categoría sistemas de gestión y estrategias son: habilidades gerenciales, planeación, gestión de materiales y equipos, y control de calidad; y en la categoría mano de obra: experiencia, actividades de entrenamiento, educación, motivación y antigüedad.

De otro lado, los resultados de Kazaz y Ulubeyli (2007) indican que los factores económicos ya no son las únicas cuestiones para tener en cuenta en la mejora de la PL en el sector de la construcción en países en desarrollo, a pesar de que los factores monetarios siguen siendo preeminentes en influir en la productividad de los empleados, los factores sociopsicológicos parecen ser cada vez más importantes como factor determinante de la PL. Los factores económicos determinantes de la PL, en orden de importancia relativa son: puntualidad de remuneración, cuantía de la remuneración, seguridad social, pago de incentivos, seguridad en el empleo y afiliación sindical; los factores sociopsicológicos incluyen: disciplina de trabajo, condiciones de salud y seguridad, satisfacción en el trabajo, desarrollo de competencias, relaciones con los compañeros de trabajo, responsabilidad, compartir problemas y su solución, oportunidades de actividad social, diferencias culturales, participación de los trabajadores en la toma de decisiones, distancia a casa y distancia a centros poblados.

Jarkas et al. (2012) identificaron como factores significativos de la productividad de los operarios en el sector de la construcción los siguientes: habilidad de la mano de obra, escasez de materiales, supervisión del trabajo, escasez de mano de obra experimentada, comunicación entre administración y operarios, falta de liderazgo de los supervisores, épocas de alta temperatura, demoras en responder a solicitudes de información, falta de suministro de trabajo con el transporte y proporción del trabajo subcontratado. Sin embargo, Naoum (2016), quien estudia la PL en el sector de la construcción, plantea que se necesitan más investigaciones para establecer la relación entre PL y los conceptos modernos de optimización del rendimiento, como cadena de suministro, lean construcción e ingeniería de valor. Estos conceptos, están dirigidos a eliminar el desperdicio, minimizar costos de transacción, así como a la mejora y transferencia de conocimientos y experiencia entre todas las partes. De otro lado, con la mirada en el sector confecciones Kaur et al. (2016) desarrollaron un estudio con el objetivo de generar conciencia sobre el impacto positivo de la implementación del lean como la solución definitiva que podría conducir a la industria global de prendas de vestir hacia el logro de la excelencia empresarial en la competencia de hoy en día.

El estudio desarrollado por Islam y Shazali (2011) genera evidencia cuantitativa de las variables de impacto en la productividad de las industrias con mano de obra intensiva, centrado en el sector de prendas de vestir; se identifica que la productividad está positivamente asociada con el ambiente de trabajo favorable, además se halla una correlación significativamente positiva entre el gasto en I+D y la productividad. De igual forma Ikram y Su (2015) centrados en el sector de confecciones, indagaron los determinantes de la productividad en pymes de Lahore, Pakistán; se identificaron como determinantes: la ambición y la motivación de los empresarios, la edad de la empresa, la instalación de la unidad del generador eléctrico, las facilidades del banco, y la gestión del capital de trabajo, se encontró que la productividad está asociada positivamente con la edad de la pyme. Otra área de estudio de la PL corresponde a la industria manufacturera. Desde allí, Aiello y Ricotta (2016) tras investigar sobre los factores que explican la variación de la productividad total de los factores en empresas manufactureras de siete países de la Unión Europea plantearon que las diferencias en la productividad se deben a características firmes, pero no observables de la empresa. Además, en cuanto al papel de la localización, demostraron que el efecto del país es más influyente que el de la región en la explicación de la productividad.

En España se identifica la formación como determinante de la PL en la industria manufacturera. Las empresas con mayor probabilidad de invertir en la formación del personal son aquellas con más trabajadores, mayor nivel de cualificación de los empleados, relaciones laborales más estables, alta participación del capital extranjero, nivel superior de capacitación tecnológica, alto nivel de asociaciones tecnológicas con otras organizaciones y actividad innovadora más intensa (Batalla-Busquets y Myrthianos, 2015). En esta misma línea, al evaluar el impacto de las variables del capital humano sobre la PL en la industria manufacturera de Malasia se concluye que el nivel de educación alcanzado por los empleados influye de forma significativa en la PL; las industrias manufactureras podrían mejorar su posición competitiva aumentando su mano de obra altamente calificada (Yunus et al., 2014). 
Fahed-Sreih (2012) examina los factores de mejora de la PL en diversos sectores del Líbano, y determinan que los factores determinantes son: delegación, decisión mediante empoderamiento, decisiones en conjunto y comportamiento de los empleados. La delegación es la variable más significativa en este estudio.

La mejora de la productividad debe ser un trabajo permanente en las empresas, Satish et al. (2013) encontró que existe una correlación mayor entre el crecimiento de la productividad y los programas de gestión (programas que cambian el método de trabajo), que con las prácticas de trabajo actuales. Esto significa que, si las empresas han aplicado la mayoría de las prácticas de trabajo modernas y han logrado grandes resultados iniciales, su mejora de la PL disminuye en otro momento si no hacen otro cambio radical y exitoso. De otro lado, el análisis de los determinantes de la PL muestra que la innovación tecnológica, las innovaciones organizativas y las tecnologías de información y comunicación, en su orden, tienen impacto en los niveles de PL en pymes (Davide et al., 2010). Sin embargo; desde la dinámica de sistemas, se plantea que los factores determinantes de la productividad están interrelacionados de forma compleja, por lo que las investigaciones tienden a concentrarse en los efectos de uno de estos factores y no explican el efecto de todos los factores influyentes. La productividad del trabajo está influenciada por varios factores que tienen interacciones complejas entre sí (Nasirzadeh y Nojedehi, 2013). En fin, una cuestión concomitante frente a la productividad es ¿qué factores son más importantes en qué sectores? (Syverson, 2011). Esto ratifica la necesidad de estudiar para cada sector los determinantes de la productividad, para este caso en las pymes de confecciones del Área Metropolitana de Bucaramanga, y aportar desde la academia al sector confecciones.

\section{METODOLOGÍA}

El proceso de análisis estadístico consistió en reducir la dimensionalidad de la matriz de datos a partir del análisis factorial exploratorio, obteniendo un conjunto de factores no correlacionados entre sí que representan la estructura interna de las variables originales.

La población objeto de estudio corresponde a las pymes del sector confecciones del AMB; según datos de la Cámara de Comercio de Bucaramanga a agosto de 2016 el número de empresas de este sector según su tamaño es de 1526 microempresas $(97,07 \%)$, 39 pequeñas empresas $(2,48 \%)$ y 7 medianas empresas $(0,45 \%)$; no se cuenta con grandes empresas.

A partir de la población conformada por 46 pymes se seleccionó la muestra de empresas participantes que representan, la unidad de análisis. Al tratarse de una población finita, el tamaño de la muestra se determina por la ecuación (1).

$$
\mathrm{n}=\frac{\mathrm{N}^{*} \mathrm{p}^{*} \mathrm{q}}{\left(\mathrm{E}^{2} / \mathrm{Z}^{2}\right)^{*}(\mathrm{~N}-1)+\mathrm{p}^{*} \mathrm{q}}
$$

Donde, n: tamaño de la muestra; Z: desviación del valor medio para obtener el nivel de confianza deseado (1,96 con un nivel de confianza de 95\%); N: tamaño de la población, 46; p: proporción esperada 0,5; q: 1-p; y, E: margen de error máximo permitido, $6 \%$. Utilizando muestreo aleatorio simple se determinó como tamaño muestral 39 empresas, las cuales fueron seleccionadas a partir del listado de pymes del AMB adquirido en la Cámara de Comercio. El método de recolección de información fue la encuesta; la construcción de ésta se alimentó de los principales hallazgos de la revisión literaria, y de entrevistas con fuentes primarias conocedoras del sector.

El planteamiento teórico sobre el cual se fundamentó la encuesta considera la PL como un constructo compuesto por dos dimensiones: la dimensión humana y la dimensión relacionada con el proceso productivo; cada una de ellas con las subdimensiones que se muestran en la Figura 1. La revisión de literatura sobre los determinantes de la productividad fue la base para la construcción del instrumento de recolección de información. Cada subdimensión está compuesta por afirmaciones planteadas a partir de elementos teóricos identificados. La Tabla 2 contiene los lineamientos generales asociados a las dimensiones, los cuales fueron explorados en las pymes en estudio.

Se entrevistaron expertos del sector a nivel local, como el director de la Asociación Colombiana de pequeños industriales del capítulo Santander, la directora de Centro de Desarrollo Productivo de Confecciones del AMB, la Coordinadora de la Feria EIMI de versiones anteriores, y dos ingenieros asesores y consultores de trayectoria en el sector. Las entrevistas buscaban conocer la estructura del sector, las principales estrategias o enfoques aplicados en las empresas para la mejora de la productividad, la percepción frente a los principales desafíos y logros en materia de productividad, entre otros aspectos. La validación del cuestionario contó con la participación de expertos del sector, entrevistados en la fase anterior, y desde el ámbito académico con la participación de seis doctores en economía, administración y áreas afines; a quienes se les solicitó la revisión 
del cuestionario preliminar. Se consolidaron las observaciones y modificaciones para llegar a una versión inicial aprobada por los expertos; la cual se aplicó como prueba piloto a dos empresarios, posteriormente se realizaron los ajustes. Realizado este proceso se contó con la versión del cuestionario a aplicar en la muestra objeto de estudio, la encuesta fue respondida por el gerente o jefe de producción. Los datos recolectados fueron procesados con el software SPSS Versión 23, arrojando los resultados que se presentan en la siguiente sección.

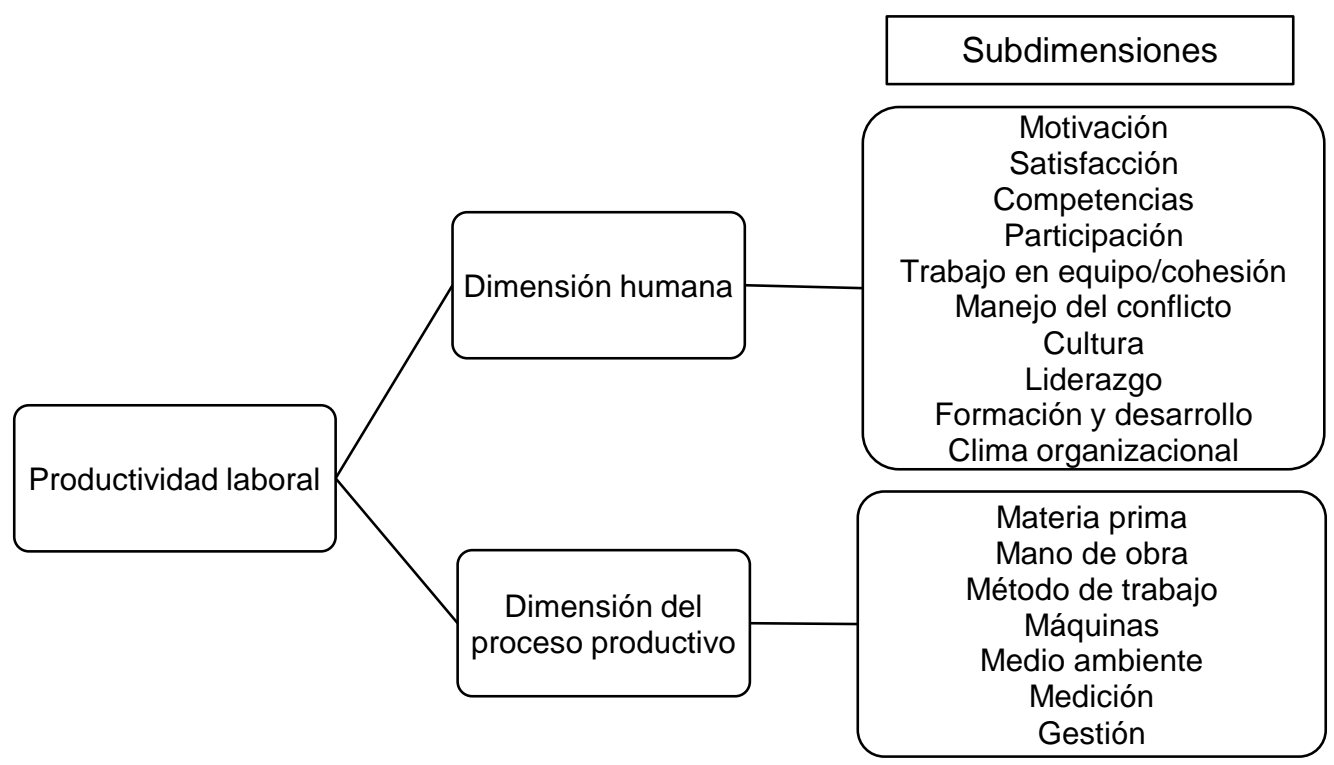

Fig. 1: Modelo conceptual

Tabla 2: Subdimensiones del modelo teórico

\begin{tabular}{|c|c|}
\hline Dimensión humana & Dimensión asociada al proceso productivo \\
\hline $\begin{array}{l}\text { Motivación: aspectos asociados a reconocimiento, comunicación, } \\
\text { compromiso con el trabajo y responsabilidad. }\end{array}$ & $\begin{array}{l}\text { Materia prima: incluye la funcionalidad, } \\
\text { disponibilidad y calidad de la materia prima. }\end{array}$ \\
\hline $\begin{array}{l}\text { Satisfacción laboral: ante el logro de objetivos, beneficios y } \\
\text { retribuciones económicas y cargas de trabajo. }\end{array}$ & $\begin{array}{l}\text { Mano de obra: abarca habilidades, incentivos y } \\
\text { capacitación. }\end{array}$ \\
\hline $\begin{array}{l}\text { Competencias: incluye el nivel de competencias, identificación de } \\
\text { competencias requeridas, desarrollo y evaluación de } \\
\text { competencias, inversión en el desarrollo de capacidades. }\end{array}$ & $\begin{array}{l}\text { Método de trabajo: explora sobre estandarización } \\
\text { de procesos, distribución de operaciones, } \\
\text { mejoras a métodos de trabajo. }\end{array}$ \\
\hline $\begin{array}{l}\text { Participación: comprende el nivel de participación en las } \\
\text { decisiones, existencia de espacios de participación, mecanismos } \\
\text { de consulta y aportes de mejora. }\end{array}$ & $\begin{array}{l}\text { Máquinas: comprende aspectos de la vida útil, } \\
\text { disponibilidad, mantenimiento y adquisición de } \\
\text { maquinaria. }\end{array}$ \\
\hline $\begin{array}{l}\text { Trabajo en equipo/Cohesión: considera las relaciones de trabajo, } \\
\text { visión común, cooperación y aporte de los empleados a equipos de } \\
\text { trabajo. }\end{array}$ & $\begin{array}{l}\text { Medio ambiente: asociado con seguridad, orden } \\
\text { y limpieza, iluminación, tranquilidad y mobiliario. }\end{array}$ \\
\hline $\begin{array}{l}\text { Manejo del conflicto: abarca solución de conflictos, creatividad para } \\
\text { resolver conflictos, mecanismos de negociación y visión del } \\
\text { conflicto como oportunidad de mejora. }\end{array}$ & $\begin{array}{l}\text { Medición: abarca registros de producción, } \\
\text { análisis de sobretiempos, controles de calidad y } \\
\text { análisis de registros de procesos. }\end{array}$ \\
\hline $\begin{array}{l}\text { Cultura organizacional: indaga sobre valores organizacionales, } \\
\text { flexibilidad en los procesos, relación entre trabajo individual y } \\
\text { objetivos organizacionales y visión compartida. }\end{array}$ & $\begin{array}{l}\text { Gestión: se relaciona con la dirección, delegación } \\
\text { de autoridad, orientación a la calidad y proceso } \\
\text { de mejora continua. }\end{array}$ \\
\hline $\begin{array}{l}\text { Liderazgo: se asocia con el reto constante a empleados, incentivos } \\
\text { por aportes a la mejora de procesos, responsabilidad ante } \\
\text { consecuencias y estilo de liderazgo. }\end{array}$ & \\
\hline $\begin{array}{l}\text { Formación y desarrollo: considera oportunidades de desarrollo, } \\
\text { formación a partir de identificación de brechas, formación en áreas } \\
\text { no técnicas y capacidades como fuente de ventaja competitiva. }\end{array}$ & \\
\hline $\begin{array}{l}\text { Clima organizacional: explora si los cargos están acordes a } \\
\text { conocimientos, retroalimentación, comunicación que incentiva el } \\
\text { logro y conocimiento de directrices organizacionales. }\end{array}$ & \\
\hline
\end{tabular}




\section{RESULTADOS Y DISCUSIÓN}

Se determinó el índice de confiabilidad, mediante el coeficiente Alfa de Cronbach, con valor 0,939, lo cual verifica una alta consistencia interna entre los factores analizados, es decir, hay una alta correlación entre cada una de las preguntas que conforman el instrumento; por lo que se puede concluir que no se perciben distorsiones que sean imputados a defectos del instrumento (George y Mallery, 2003). Se realizaron dos análisis factoriales exploratorios: uno asociado a la dimensión humana y el otro al proceso productivo de la empresa, con los resultados plasmados a continuación.

\section{Análisis factorial exploratorio de la dimensión humana}

El análisis factorial exploratorio pretende hallar un nuevo conjunto de variables (factores), menor en número que las variables originales, que exprese lo que es común entre ellas. En este sentido, se verificó que éste es adecuado para el conjunto de datos de la dimensión humana, ya que cumple los criterios necesarios para el mismo: 1) correlaciones moderadas como se observa en la matriz de correlaciones en la Tabla 3; 2) prueba de esfericidad de Bartlett (chi-cuadrado= 213,04; $p$-value $<0,05$ ); 3) determinante de la matriz de correlación 0,02 (próximo a cero) y; 4) grado de adecuación de los datos, medido a partir del estadístico de Kaiser-Meyer-Olkin $(0,73)$. En virtud de la baja correlación de la variable competencias con las demás, se excluyó del modelo.

Tabla 3: Matriz de correlaciones de la dimensión humana

\begin{tabular}{|c|c|c|c|c|c|c|c|c|c|c|c|}
\hline & & $\begin{array}{l}\text { Motiva- } \\
\text { ción }\end{array}$ & $\begin{array}{c}\text { Satis- } \\
\text { facción }\end{array}$ & $\begin{array}{c}\text { Compe- } \\
\text { tencia }\end{array}$ & $\begin{array}{l}\text { Partici- } \\
\text { pación }\end{array}$ & \begin{tabular}{|c|} 
Cohe- \\
sión \\
\end{tabular} & $\begin{array}{l}\text { Con- } \\
\text { flicto }\end{array}$ & \begin{tabular}{|c|} 
Cultu- \\
ra
\end{tabular} & $\begin{array}{l}\text { Lide- } \\
\text { razgo }\end{array}$ & $\begin{array}{l}\text { Forma- } \\
\text { ción }\end{array}$ & Clima \\
\hline \multirow{2}{*}{ Motivación } & Correlación & 1 & & & & & & & & & \\
\hline & p-value & & & & & & & & & & \\
\hline \multirow{2}{*}{$\begin{array}{l}\begin{array}{l}\text { Satisfacción } \\
\text { laboral }\end{array} \\
\end{array}$} & Correlación & 0,584 & 1 & & & & & & & & \\
\hline & $p$-value & 0,000 & & & & & & & & & \\
\hline \multirow{2}{*}{ Competencia } & Correlación & 0,177 & 0,178 & 1 & & & & & & & \\
\hline & $p$-value & 0,082 & 0,079 & & & & & & & & \\
\hline \multirow{2}{*}{ Participación } & Correlación & 0,510 & 0,295 & 0,301 & 1 & & & & & & \\
\hline & $p$-value & 0,001 & 0,068 & 0,053 & & & & & & & \\
\hline \multirow{2}{*}{$\begin{array}{l}\text { Trabajo en } \\
\text { equipo/cohesión }\end{array}$} & Correlación & 0,585 & 0,625 & 0,212 & 0,549 & 1 & & & & & \\
\hline & $p$-value & 0,000 & 0,000 & 0,096 & 0,000 & & & & & & \\
\hline \multirow{2}{*}{\begin{tabular}{|l|}
$\begin{array}{l}\text { Manejo del } \\
\text { conflicto }\end{array}$ \\
\end{tabular}} & Correlación & 0,615 & 0,515 & 0,289 & 0,506 & 0,600 & 1 & & & & \\
\hline & $\mathrm{p}$-value & 0,000 & 0,001 & 0,045 & 0,001 & 0,000 & & & & & \\
\hline \multirow{2}{*}{\begin{tabular}{|l|}
$\begin{array}{l}\text { Cultura } \\
\text { organizacional }\end{array}$ \\
\end{tabular}} & Correlación & 0,456 & 0,453 & 0,195 & 0,695 & 0,530 & 0,469 & 1 & & & \\
\hline & $p$-value & 0,004 & 0,004 & 0,065 & 0,000 & 0,001 & 0,003 & & & & \\
\hline \multirow{2}{*}{ Liderazgo } & Correlación & 0,587 & 0,463 & 0,147 & 0,718 & 0,533 & 0,434 & 0,690 & 1 & & \\
\hline & $\mathrm{p}$-value & 0,000 & 0,003 & 0,047 & 0,000 & 0,000 & 0,006 & 0,000 & & & \\
\hline \multirow{2}{*}{$\begin{array}{l}\text { Formación y } \\
\text { desarrollo }\end{array}$} & Correlación & 0,235 & 0,284 & 0,578 & 0,395 & 0,343 & 0,536 & 0,301 & 0,522 & 1 & \\
\hline & $p$-value & 0,094 & 0,080 & 0,000 & 0,013 & 0,032 & 0,000 & 0,063 & 0,001 & & \\
\hline \multirow{2}{*}{\begin{tabular}{|l|} 
Clima \\
organizacional
\end{tabular}} & Correlación & 0,316 & 0,656 & 0,258 & 0,374 & 0,541 & 0,477 & 0,430 & 0,483 & 0,432 & 1 \\
\hline & $\mathrm{p}$-value & 0,050 & 0,000 & 0,091 & 0,019 & 0,000 & 0,002 & 0,006 & 0,002 & 0,006 & \\
\hline
\end{tabular}

El análisis es apropiado ya que el $62,32 \%$ de la variabilidad total es explicada por el modelo factorial. Para lograr una mejor interpretación de las variables subyacentes a los diferentes factores, se usó el método de extracción Alfa y se rotó la matriz de cargas factoriales según la técnica Promax, obteniendo la matriz de la Tabla 4.

Tabla 4: Matriz Factorial Rotada, dimensión humana

\begin{tabular}{|c|c|c|}
\hline \multirow{2}{*}{ Subdimensión } & \multicolumn{2}{|c|}{ Factor } \\
\hline & Comportamiento grupal ${ }^{1}$ & Ambiente social de trabajo ${ }^{2}$ \\
\hline Motivación & 0,568 & $0,580^{2}$ \\
\hline Satisfacción laboral & 0,453 & $0,916^{2}$ \\
\hline Participación & $0,846^{1}$ & 0,408 \\
\hline Trabajo en equipo/cohesión & 0,622 & $0,745^{2}$ \\
\hline Manejo del conflicto & $0,688^{1}$ & 0,460 \\
\hline Cultura organizacional & $0,649^{1}$ & 0,554 \\
\hline Liderazgo & $0,770^{1}$ & 0,691 \\
\hline Formación y desarrollo & $0,475^{1}$ & 0,292 \\
\hline Clima organizacional & 0,399 & $0,628^{2}$ \\
\hline
\end{tabular}


Cada variable va a representar el factor donde su carga factorial sea mayor, se observa el superíndice 1 para las variables del factor denominado comportamiento grupal, y 2 para el factor ambiente social de trabajo. El nombre dado a los factores se establece teniendo en cuanta las variables que representan, es así como al factor representado por las variables: participación, manejo del conflicto, cultura organizacional, liderazgo y formación y desarrollo se le da el nombre de comportamiento grupal dado que estas variables hacen referencia principalmente a elementos propios de la interacción con los otros o de la colectividad.

Las variables: motivación, satisfacción laboral, cohesión, y clima organizacional representan el factor llamado ambiente social de trabajo, nombre seleccionado considerando que el ambiente social hace referencia a interacciones entre los individuos y las características del entorno, así como lo percibido por las personas, y se agrega de trabajo porque dicho ambiente social está en un contexto laboral. Dichos factores no están correlacionados entre sí; la figura 2 muestra los factores de la dimensión humana de la productividad laboral: comportamiento grupal y ambiente social de trabajo, y las variables que los representan.

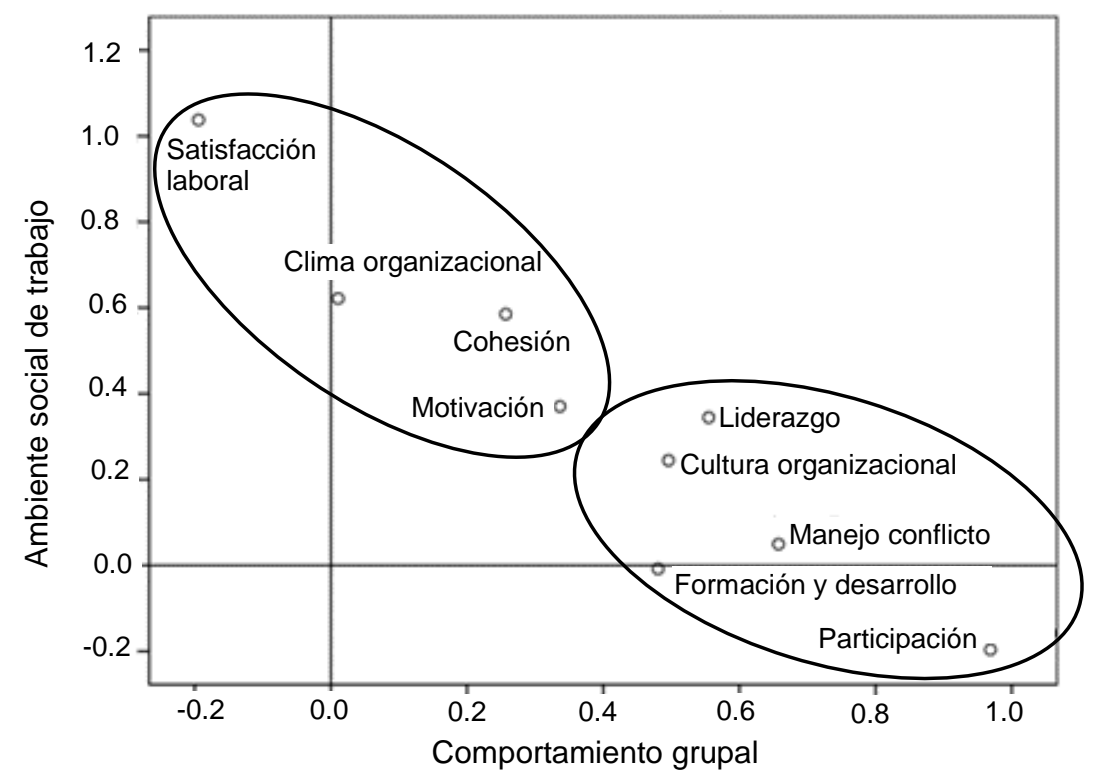

Fig.2: Gráfico Factorial de la dimensión humana.

Los valores de los dos factores resultantes se obtienen de la matriz de puntuaciones factoriales de los componentes, presentada en la Tabla 5.

Tabla 5: Puntuaciones Factoriales de la dimensión humana

\begin{tabular}{|l|c|c|}
\hline \multirow{2}{*}{ Subdimensión } & \multicolumn{2}{|c|}{ Factor } \\
\cline { 2 - 3 } & Comportamiento grupal & Ambiente social de trabajo \\
\hline Motivación & $-0,241$ & 0,035 \\
Satisfacción laboral & $-0,174$ & 0,655 \\
Participación & 0,464 & $-0,003$ \\
Cohesión & 0,142 & 0,187 \\
Conflicto & 0,469 & 0,004 \\
Cultura organizacional & $-0,106$ & 0,132 \\
Liderazgo & 0,587 & 0,077 \\
Formación y desarrollo & $-0,167$ & 0,014 \\
Clima organizacional & 0,149 & 0,046 \\
\hline
\end{tabular}

Se tiene que los factores identificados tienen las expresiones dadas en las ecuaciones (2) y (3). 
Ambiente social de trabajo $=0.035$ Motivación+0.655Satisfacción laboral-0.003Participación+0.187Cohesión+

0.004Conflicto+0.132Cultura org.+0.077Liderazgo+0.014Formación+0.046Clima org

\section{Análisis factorial exploratorio de la dimensión asociada al proceso productivo}

De igual manera que para la dimensión humana se verificó que el análisis factorial exploratorio es adecuado para el conjunto de datos de la dimensión asociada al proceso productivo, encontrando que se cumplen los criterios mencionados anteriormente: 1) correlaciones moderadas en la matriz de correlación, como se observa en la Tabla 6; 2) prueba de esfericidad de Bartlett (chi-cuadrado=45,994; $p$-value $<0,05$ ), 3) determinante de la matriz de correlación 0,26 (próximo a cero) y, 4) grado de adecuación de los datos, medida a partir del estadístico de Kaiser-Meyer-Olkin $(0,557)$. El análisis de la matriz de correlaciones determina eliminar la variable materia prima por su poca correlación con las demás variables.

Tabla 6: Matriz de Correlaciones, referente al proceso productivo

\begin{tabular}{|c|c|c|c|c|c|c|c|c|}
\hline & & $\begin{array}{c}\text { Materia } \\
\text { Prima }\end{array}$ & $\begin{array}{c}\text { Mano de } \\
\text { Obra }\end{array}$ & $\begin{array}{c}\text { Método de } \\
\text { Trabajo }\end{array}$ & Máquinas & $\begin{array}{c}\text { Medio } \\
\text { Ambiente }\end{array}$ & Medición & Gestión \\
\hline Materia Prima & $\begin{array}{l}\text { Correlación } \\
\text { p-value }\end{array}$ & 1 & & & & & & \\
\hline Mano de Obra & $\begin{array}{l}\text { Correlación } \\
\text { p-value }\end{array}$ & $\begin{array}{l}0,203 \\
0,092\end{array}$ & 1 & & & & & \\
\hline $\begin{array}{l}\text { Método de } \\
\text { Trabajo }\end{array}$ & $\begin{array}{l}\text { Correlación } \\
\text { p-value }\end{array}$ & $\begin{array}{l}0,404 \\
0,011\end{array}$ & $\begin{array}{l}0,380 \\
0,017\end{array}$ & 1 & & & & \\
\hline Máquinas & $\begin{array}{l}\text { Correlación } \\
\text { p-value }\end{array}$ & $\begin{array}{l}-0,078 \\
0,096\end{array}$ & $\begin{array}{l}0,535 \\
0,000\end{array}$ & $\begin{array}{l}0,343 \\
0,033\end{array}$ & 1 & & & \\
\hline $\begin{array}{l}\text { Medio } \\
\text { Ambiente }\end{array}$ & $\begin{array}{l}\text { Correlación } \\
\text { p-value }\end{array}$ & $\begin{array}{l}0,135 \\
0,094\end{array}$ & $\begin{array}{l}0,436 \\
0,005\end{array}$ & $\begin{array}{l}0,332 \\
0,039\end{array}$ & $\begin{array}{l}0,366 \\
0,022\end{array}$ & 1 & & \\
\hline Medición & $\begin{array}{l}\text { Correlación } \\
\text { p-value }\end{array}$ & $\begin{array}{l}0,119 \\
0,095\end{array}$ & $\begin{array}{l}0,468 \\
0,003\end{array}$ & $\begin{array}{l}0,452 \\
0,004\end{array}$ & $\begin{array}{l}0,315 \\
0,051\end{array}$ & $\begin{array}{l}0,130 \\
0,430\end{array}$ & 1 & \\
\hline Gestión & $\begin{array}{l}\text { Correlación } \\
\text { p-value }\end{array}$ & $\begin{array}{l}0,297 \\
0,067\end{array}$ & $\begin{array}{l}0,435 \\
0,006\end{array}$ & $\begin{array}{l}0,608 \\
0,000\end{array}$ & $\begin{array}{l}0,208 \\
0,092\end{array}$ & $\begin{array}{l}0,301 \\
0,063\end{array}$ & $\begin{array}{l}0,320 \\
0,047\end{array}$ & 1 \\
\hline
\end{tabular}

Al verificar la factibilidad del análisis factorial exploratorio realizado se determina que es apropiado, el 59,42\% de la variabilidad total es explicada en los dos factores del modelo factorial obtenido; en este sentido, se extrajeron los factores con el método Alfa y se rotó la matriz de cargas factoriales según la técnica Promax Kappa obteniendo la matriz de la Tabla 7. Nuevamente se asignan las subdimensiones al factor donde su carga factorial es mayor; el superíndice 3 acompaña las cargas factoriales de las subdimensiones que representan al factor gestión de procesos y el 4 , a aquellas que representan el factor capacidades/gestión.

Tabla 7: Matriz Factorial Rotada, referente al proceso productivo

\begin{tabular}{|l|c|c|}
\hline \multirow{2}{*}{ Subdimensión } & \multicolumn{2}{|c|}{ Factor } \\
\cline { 2 - 3 } & Gestión de Procesos & Capacidades / control \\
\hline Mano de Obra & 0,341 & $0,757^{4}$ \\
Método de Trabajo & $0,680^{3}$ & 0,485 \\
Máquinas & 0,215 & $0,595^{4}$ \\
Medio Ambiente & $0,460^{3}$ & 0,353 \\
Medición & 0,189 & $0,401^{4}$ \\
Gestión & $0,875^{3}$ & 0,213 \\
\hline
\end{tabular}

El primer factor fue denominado gestión del proceso por su asociación con: método de trabajo, medio ambiente y gestión; el segundo factor fue llamado capacidades/control ya que está conformado por las variables: mano de obra; máquinas y medición. Las cargas factoriales presentadas en la Tabla 7 permiten diferenciar dos factores: gestión del proceso y capacidades/control, los cuales agrupan las variables mencionadas como se ve en los agrupamientos de la Figura 3. 


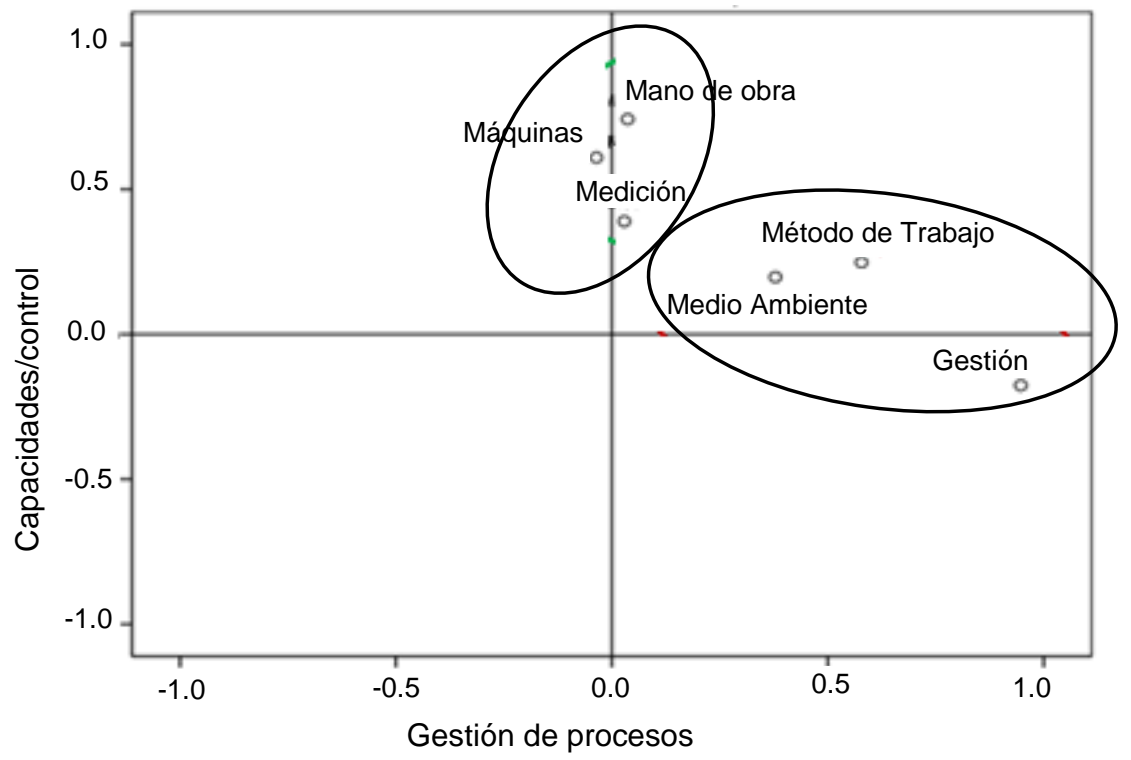

Fig. 3: Gráfico Factorial dimensión del proceso productivo.

La Tabla 8 presenta los valores de las puntuaciones de los factores resultantes asociados a la dimensión del proceso productivo, de tal forma que estos tienen las expresiones dadas en las ecuaciones (4) y (5).

Tabla 8: Puntuaciones factoriales, dimensión asociada al proceso productivo

\begin{tabular}{|l|c|c|}
\hline \multirow{2}{*}{ Subdimensión } & \multicolumn{2}{|c|}{ Factor } \\
\cline { 2 - 3 } & Gestión de Procesos & Capacidades/Control \\
\hline Mano de obra & 0,110 & 0,670 \\
Método Trabajo & 0,280 & 0,433 \\
Máquinas & 0,114 & 0,251 \\
Medio Ambiente & 0,013 & $-0,095$ \\
Medición & $-0,081$ & $-0,017$ \\
Gestión & 0,707 & $-0,138$ \\
\hline
\end{tabular}

Gestión de procesos=0,110 Mano de obra+0,280 Método de trabajo+0,114 Máquinas +0,013 Medio Ambiente-0,081 Medición+0,707 Gestión

Capacidades/control=0,670 Mano de obra+0,433 Método de trabajo+0,251 Máquinas -0,095 Medio Ambiente-0,017 Medición-0,138 Gestión

Es decir, las diecisiete subdimensiones de la encuesta correspondientes a las dimensiones humana y del proceso productivo de la PL en las pymes en estudio, fueron resumidas en cuatro: comportamiento grupal, ambiente social de trabajo; gestión de procesos y capacidades/control, cuyas estructuras algebraicas están dadas por las ecuaciones 2 a 5 . Siendo estos los factores determinantes de la productividad laboral en las pymes del sector confecciones de Bucaramanga y su área metropolitana.

\section{CONCLUSIONES}

La revisión de la literatura relacionada con la Productividad Laboral -PL- y sus determinantes, permite concluir que, a pesar de ser un tema ampliamente estudiado y aplicado en varias disciplinas, es relevante seguir aportando a su frontera de conocimiento, ya que la PL es afectada por diversos factores relacionados con las condiciones propias de la región y/o país y con el sector donde se analice, lo cual es sustentado por los autores en referencia. Por tanto, la identificación de los factores determinantes de las pymes de confecciones del Área Metropolitana de Bucaramanga -AMB- es un hallazgo valioso y pertinente al sector y la región objeto de estudio, debido a su impacto en la economía y desarrollo empresarial.

En las condiciones propias de estudio se identificó que los factores determinantes para la PL en las pymes de confecciones son: comportamiento grupal, ambiente social de trabajo, gestión del proceso y capacitación/control. Los dos primeros factores asociados a la dimensión humana de la PL y los dos últimos 
a la dimensión del proceso productivo. Desde la dimensión humana, el factor comportamiento grupal está representado por las variables: participación, manejo del conflicto, cultura organizacional, liderazgo y formación y desarrollo. Las variables: motivación, satisfacción laboral, cohesión, y clima organizacional representan el factor ambiente social de trabajo. En la dimensión relacionada con el proceso productivo el primer factor fue denominado gestión del proceso, este se asocia con: método de trabajo, medio ambiente y gestión; el segundo factor fue llamado Capacidades/control, está conformado por las variables: mano de obra; máquinas y medición.

Mantener el posicionamiento del sector confecciones en el Área Metropolitana de Bucaramanga, SantanderColombia requiere del mejoramiento de la PL. Según los hallazgos de la investigación se concluye sobre la relevancia de evaluar y propender por la mejora de los factores asociados a la dimensión humana. De otro lado, desde la dimensión del proceso productivo, es importante la adecuada gestión de los procesos, contar con las capacidades necesarias y hacer control sobre dichos procesos para favorecer la productividad del talento humano.

\section{REFERENCIAS}

Aiello, F. y F. Ricotta, Firm Heterogeneity in Productivity across Europe: Evidence from Multilevel Models, doi: 10.1080/10438599.2015.1057001, Economics of Innovation and New Technology, 25(1), 57-89 (2016)

Batalla-Busquets, J.M. y V. Myrthianos, The Impact of Innovation and the Use of ICTs on Human Capital Development in Spanish Industry, doi:10.3926/ic.423, Intangible Capital, 11(2), 249-269 (2015)

Cámara de Comercio de Bucaramanga. Consulta Compite 360, Bucaramanga, Colombia (2017)

Cardozo, E., C. Rodríguez y W. Guaita, Las Pequeñas y Medianas Empresas Agroalimentarias en Venezuela y el Desarrollo Sustentable: Enfoque basado en los Principios de Manufactura Esbelta, doi: 10.4067/S071807642011000500006, Información Tecnológica, 22(5), 39-48 (2011)

Cequea, M. y C. Rodríguez-Monroy, Productividad y Factores Humanos, Un modelo con Ecuaciones Estructurales, ISSN Electrónico: 2244-7776, Interciencia, 37(2), 121-127 (2012)

Chadwick, C., S. Way, G. Kerr y J. Thacker, Boundary Conditions of the High-Investment Human Resource SystemsSmall-Firm Labor Productivity Relationship, doi: 10.1111/peps.12015, Personnel Psychology, 66(2), $311-343$ (2013)

Congreso de la República, Ley 905 de 2004, por medio de la cual se modifica la Ley 590 de 2000, Diario Oficial No. 45.628, Bogotá, Colombia (2004)

Consejo Privado de Competitividad, Informe Nacional de Competitividad 2016-2017, ISSN: 2016- 1430, Informes Nacionales por año (2016)

DANE, Boletín técnico Encuesta Anual Manufacturera - EAM 2015, Estadísticas por Tema Industria (2016)

Davide, A., M. Mazzanti y P. Pini, Productivity, Innovation Strategies and Industrial Relations in SMEs. Empirical Evidence for a Local Production System in Northern Italy, doi: 10.1080/02692171.2010.483790, International Review of Applied Economics, 24(4), 453-482 (2010)

Fahed-Sreih, J., The Effect of Investing in Hiring, Human Resource Planning and Employee Development on Labor Productivity: Case of Lebanon, Journal of International Business Research, 11(1), 29-51 (2012)

George, D. y M. Mallery, Using SPSS for Windows Step by Step: a Simple Guide and Reference, Allyn \& Bacon, Boston, Estados Unidos (2003)

Hernández, H.G., D. Cardona y J. Del Rio, Direccionamiento Estratégico: Proyección de la Innovación Tecnológica y Gestión Administrativa en las Pequeñas Empresas, doi: 10.4067/S0718-07642017000500003, Información Tecnológica, 28(5), 15-22 (2017)

Ikram, A. y Q. Su, Determinants of Productivity in the Ready-made Garments SMEs of Lahore, Pakistan, doi: 10.2991/97894-6239-100-0, Proceedings of the 5th International Asia Conference on Industrial Engineering and Management Innovation (IEMI2014), 81-86, Paris, Francia (2015)

Islam, S. y S. Shazali, Determinants of Manufacturing Productivity: Pilot Study on Labor-Intensive Industries, doi: 10.1108/17410401111150751, International Journal of Productivity and Performance Management, 60(6), 567-582 (2011)

Jarkas, A. M., C. Kadri y J. Younes, A Survey of Factors Influencing the Productivity of Construction Operatives in the State of Qatar, doi:10.1080/15623599.2012.10773192, International Journal of Construction Management, 12(3), 1-23 (2012)

Kaur, P., K. Marriya y R. Kashyap, Assessment of Lean in Apparel Export Industry of National Capital Region (India), ISSN: 1533-0915, Journal of Textile and Apparel, Technology and Management, 10(1), 1-17 (2016)

Kazaz, A. y S. Ulubeyli, Drivers of Productivity among Construction Workers: A Study in a Developing Country, doi: 10.1016/j.buildenv.2006.04.020, Building and Environment, 42(5), 2132-2140 (2007)

Mathur, A., M. Mittal y G. Dangayach, Improving Productivity in Indian SMEs, doi: 10.1080/09537287.2011.642150, Production Planning \& Control, 23(10-11), 754-768 (2012) 
Ministerio de Comercio, Industrial y Turismo, Así avanza la Transformación Productiva 2013, Programa de Transformación Productiva, Bogotá, Colombia (2013)

Naoum, S.G., Factors Influencing Labor Productivity on Construction Sites: A State-of-the-Art Literature Review and a Survey, doi: 10.1108/IJPPM-03-2015-0045, International Journal of Productivity and Performance Management, 65(3), 401-421 (2016)

Nasirzadeh, F. y P. Nojedehi, Dynamic Modeling of Labor Productivity in Construction Projects, doi: 10.1016/j.jproman.2012.11.003, International Journal of Project Management, 31(6), 903-911 (2013)

Oficina Regional de la OIT para América Latina y el Caribe, Panorama Laboral 2012. América Latina y el Caribe, Organización Internacional del Trabajo, Lima, Perú (2012)

Parida, P.C. y K. Pradhan, Productivity and Efficiency of Labour Intensive Manufacturing Industries in India: An Empirical Analysis, doi: 10.1108/IJDI-12-2015-0081, International Journal of Development Issues, 15(2), 130-152 (2016)

Porter, M. E., Ser competitivo, 163-202, Deusto, Barcelona, España (2009)

Putnik, G.D., A. Alves, J. Dinis-Carvalho y R. Sousa, Lean Production as Promoter of Thinkers to Achieve Companies' Agility, doi: 10.1108/09696471211219930, The Learning Organization, 19(3), 219-237 (2012)

Rojas, E.M. y P. Aramvareekul, Labor Productivity Drivers and Opportunities in the Construction Industry, doi: 10.1061/(ASCE)0742-597X(2003)19:2(78), Journal of Management in Engineering, 19(2), 78-82 (2003)

Satish, U., L. Cleckner y J. Vasselli, Impact of VOCs on Decision Making and Productivity, doi: 10.1080/17508975.2013.812956, Intelligent Buildings International, 5(4), 213-220 (2013)

Smith, A., The wealth of nations, 97-102. Alianza Editorial, Ed., Londres, Reino Unido (1776)

Sumanth, D., Ingeniería y Administración de la Productividad, McGraw-Hill, México D.F., México (1996)

SURA, Análisis de Comportamiento y Oportunidades del Sector Sistema Moda, Bogotá D.C., Colombia (2014)

Syverson, C., What Determines Productivity?, doi: 10.1257/jel.49.2.326, J. of Economic Literature, 49(2), $326-365$ (2011)

Yang, T.M. y C. Su, Using a Systematic Organization Change Approach to Improve Productivity and Application with a Case Study on Semiconductor Manufacturing Company, doi: 10.6220/joq.2012.19(1).03，品質學報, 19(1), 39-58 (2012)

Yunus, N.M., R. Said y S. Law, Do cost of training, education level and R\&D investment matter towards influencing labour productivity?, ISSN: 0127-1962, Jurnal Ekonomi Malaysia, 48(1), 133-142 (2014)

Zuleta, L. y L. Jaramillo, Reestructuración y Competitividad de la Cadena Textil - Confecciones en Colombia; in El Crecimiento de la Productividad en Colombia: resultados del estudio nacional sobre determinantes del crecimiento de la productividad by Departamento Nacional de Planeación, pp. 345-373, Bogotá, Colombia (1996) 